\title{
Acromegalia: una nuova prospettiva fisiopatologica dai tessuti periferici?
}

\author{
Aart J. van der Lely ${ }^{1}$
}

Pubblicato online: 12 luglio 2017

(C) The Author(s) 2017. This article is published with open access at Springerlink.com

\begin{abstract}
Sommario La terapia medica dell'acromegalia, con analoghi della somatostatina a prolungata durata di azione (LASMSA) e con l'antagonista recettoriale dell'ormone della crescita Pegvisomant (PEGV), rende possibile il raggiungimento di normali livelli circolanti di IGF-I nella maggioranza dei pazienti acromegalici. Le due classi di farmaci, comunque, hanno un differente impatto sull' asse GH-IGF-I, il che complica la tradizionale valutazione biochimica della risposta alla terapia. In questa rassegna si postula che la terapia con LA-SMSA in certi pazienti normalizzi i livelli circolanti di IGF-I senza influenzare gli effetti dell'aumento del GH a livello di tessuti extraepatici. Tale eventualità può risultare nella persistenza dell'attività della malattia, per la quale si propone la definizione di acromegalia extraepatica. Il Pegvisomant, d'altra parte, blocca gli effetti sistemici del GH, evento che non è necessariamente e attendibilmente testimoniato dai livelli circolanti di IGF-I; inoltre, il trattamento con Pegvisomant causa ulteriore aumento dei livelli circolanti di GH. Il monitoraggio della terapia medica, basato su marcatori bioumorali tradizionali, è quindi complicato. Peraltro, il differente meccanismo d'azione di LA-SMSA e PEGV incoraggia l'impiego dei due farmaci in combinazione, rendendo necessaria una revisione critica dei
\end{abstract}

Proposto da Francesco Trimarchi ed Emanuela Arvat.

Questa rassegna è basata su un aggiornamento e una revisione del lavoro di Neggers et al., citato in [7].

Materiale elettronico supplementare La versione elettronica di questo articolo (DOI:10.1007/s40619-017-0327-x) contiene materiale supplementare, disponibile per gli utenti autorizzati.

$\square$ A.J. van der Lely

a.vanderlelij@erasmusmc.nl

1 Department of Internal Medicine, Erasmus University MC, Rotterdam, The Netherlands correnti concetti relativi al trattamento dell'acromegalia e al monitoraggio degli effetti della terapia.

Parole chiave Acromegalia A Analoghi della

somatostatina $\cdot$ Pegvisomant $\cdot$ IGF-I

\section{Introduzione}

L'acromegalia è una malattia rara generalmente causata da un tumore dell'adenoipofisi secernente ormone della crescita $(\mathrm{GH})$. I presidi terapeutici attualmente disponibili hanno lo scopo di normalizzare i livelli circolanti di IGF-I per mezzo della riduzione della ipersecrezione di GH o dell'inibizione dei suoi effetti [1]. L'efficacia dei differenti trattamenti viene monitorata misurando periodicamente e comparativamente i livelli circolanti di IGF-I, metodo più pratico rispetto al dosaggio seriato dei livelli di $\mathrm{GH}$ e tale dosaggio è applicabile anche nel paragonare gli effetti degli analoghi della somatostatina a lunga durata di azione (LA-SMSA) con quelli dell' antagonista recettoriale del GH, Pegvisomant (PEGV). Tale approccio si basa sull'ipotesi che i livelli circolanti di IGF-I riflettano in maniera univoca l'attività della malattia, ipotesi che non appare necessariamente valida.

In questa rassegna sarà esaminata la relazione reciproca GH-IGF-I con specifica attenzione alle significative differenze fra i meccanismi d'azione dei LA-SMSA e del PEGV. Grazie a tale approccio si proporrà un nuovo paradigma dell'acromegalia, epatica ed extra-epatica e se ne discuteranno le implicazioni cliniche. 


\section{Gli effetti del GH sono tessuto-specifici e concentrazione-dipendenti}

Gli effetti fisiologici del GH rispetto a quelli dell'IGF-I restano ancora non completamente chiariti. È stato difficile, in passato, differenziare gli effetti del GH e dell' IGF-I a livello tissutale in condizioni fisiologiche. Tuttavia, il fatto che il GH possegga un'attività diabetogena o "anti-insulinica" mentre l'IGF-I (come la sua denominazione indica) svolga azioni simil-insuliniche, definisce chiaramente l'esistenza di differenze fisiologiche nell'azione dei due ormoni peptidici. Il paragrafo successivo è dedicato all'analisi di studi sugli effetti specifici del GH e sulle interazioni fisiologiche GH-IGF-I, condotti in modelli animali.

\section{Studi dell'azione del GH vs. IGF-I in modelli animali}

È a tutti evidente che, essendo il GH una sostanza diabetogena, non ne può essere previsto il suo impiego terapeutico nel diabete di tipo 2, nonostante il potenziale effetto positivo della sua azione lipolitica e anti-lipogenica. Solo due studi hanno documentato effetti benefici del GH sul metabolismo del glucosio in pazienti con diabete di tipo 2. È stato realizzato un modello murino, utilizzando topi maschi C57BL/6J, per spiegare gli effetti del GH sul diabete di tipo 2 e l'obesità, indotti dalla dieta ad alto contenuto di lipidi [2]. Durante gli studi, i topi sono stati trattati con differenti dosi di $\mathrm{GH}$ valutando il peso e la composizione corporea, la glicemia a digiuno, l'insulinemia e l'IGF-I, la tolleranza al glucosio, il triacilglicerolo epatico, il peso di singoli tessuti e altri dati di laboratorio. Sono stati riportati numerosi importanti risultati: per prima cosa sono stati osservati una riduzione dose-dipendente della massa grassa e un aumento della massa magra, evidente per le dosi di GH più elevate, sebbene solo la massima dose utilizzata avesse indotto un aumento dei livelli circolanti di IGF-I. Tali risultati suggeriscono che alcuni effetti del GH siano indipendenti dai livelli circolanti di IGF-I. Secondo, l'aumento della massa magra veniva osservato prima della riduzione del tessuto adiposo bianco (WAT), indicando che gli effetti fisiologici del GH non si esercitano in maniera sincrona in tessuti differenti. Terzo, la perdita di WAT interessava specificamente il grasso sottocutaneo e mesenterico, supportando l'ipotesi che non tutti i depositi di WAT siano eguali in termini di risposta all'azione del GH. Tale evidenza è peraltro in accordo con altri dati dimostranti l'aumento dei depositi di WAT sottocutaneo in condizioni di carenza di GH e, nel complesso, sottolinea l'importanza dell'effetto del GH per se sulla composizione corporea. Si deve comunque sottolineare che le modificazioni della composizione corporea GH-dipendenti possono essere dovute ad azioni autocrine/paracrine delle IGF-I e non ad azioni dirette del GH. L'importanza della produzione autocrina o paracrina dell'IGF-I è stata, infatti, documentata in topi deficienti per lo specifico gene epatico codificante per le IGF-I.

Altri studi in modelli murini, tendenti a discriminare l'effetto del GH da quello delle IGF-I, condotti una ventina d'anni fa, hanno dimostrato che gli animali con alti livelli di GH e IGF-I sviluppavano glomerulosclerosi, al contrario dei topi con isolato aumento di IGF-I. Un'estensione di questi studi, su topi transgenici che esprimono analoghi del $\mathrm{GH}$, ha dimostrato, inoltre, che l'espressione di un analogo bovino (b) del GH, con mutazioni L121P, E126G, non induce modificazione delle dimensioni dell'animale o dei livelli di IGF-I, ma è in grado di determinare un quadro di glomerulosclerosi grave quanto quella osservata in topi wild type bGH, in accordo con l'ipotesi che il GH agisca sul rene indipendentemente dall' aumento di IGF-I. Inoltre, la nefropatia diabetica indotta da streptozotocina non si osserva in animali che esprimono un antagonista del recettore del GH o che sono trattati con PEGV. È importante il fatto che la malattia renale è prevenuta dal PEGV anche in assenza di riduzione dell' IGF-I circolante [3]. Ciò implica quindi che il GH abbia un effetto diretto sul rene, indipendente dai livelli circolanti di IGF-I.

I risultati di questi studi sull'azione di GH in modelli murini suggeriscono pertanto che il GH eserciti effetti temporali e tessuto-specifici indipendenti dall'elevazione dell'IGF-I nel siero.

\section{La relazione fra insulina portale e sensibilità epatica al GH}

L'uomo e altri mammiferi sopportano il digiuno prolungato perché sono capaci di utilizzare i grassi di deposito in assenza di pronta disponibilità di carboidrati [4]. Il digiuno prolungato si associa a una graduale riduzione della produzione epatica di IGF-I, evento di senso teleologico, in considerazione degli effetti insulino-simili dell'IGF-I. Uno studio di Ho et al. [5] suggerisce che la produzione epatica di IGF-I, indotta dal GH, sia regolata dai livelli portali di insulina. Lo studio dimostra che l'insulina promuove la traslocazione in superficie del recettore del GH (GHR). In presenza di alti livelli di insulina portale, il fegato diviene GH sensibile indipendentemente dalla causa di iperproduzione insulinica [5]. Inoltre, l'insulina portale inibisce anche la produzione della proteina di trasporto dell'IGF-I (IGFBP-1), fatto che aumenta la biodisponibilità di IGF-I.

Pertanto, elevati livelli portali di insulina aumentano la sensibilità epatica al GH (per mezzo della up-regolazione dei recettori di superficie del GH) determinando, perciò, aumento della produzione di IGF-I epatica con conseguente aumento dell'IGF-I circolante. Al contrario, bassi livelli 
di insulina portale riducono la sensibilità del fegato per il $\mathrm{GH}$ e, conseguentemente, i livelli sierici di IGF-I.

\section{Perché gli acromegalici hanno alti livelli di IGF-I}

Gli acromegalici hanno alti livelli circolanti di IGF-I in conseguenza dell'ipersecrezione del GH. L'eccesso di GH, in aggiunta, stimola la lipolisi e induce resistenza periferica agli effetti dell'insulina sul metabolismo del glucosio a livello epatico e muscolare. Il risultato finale è caratterizzato da alti livelli di glucosio, acidi grassi liberi e insulina [6]. L'iperinsulinemia indotta dal GH, a sua volta, stimola ulteriormente la produzione epatica di IGF-I e riduce i livelli di IGFBP-1. La rilevanza di questo effetto è testimoniata dall'osservazione che l'ipoinsulinemia indotta da digiuno prolungato può normalizzare completamente i livelli di IGF-I negli acromegalici. In conclusione, gli acromegalici hanno aumentati livelli di IGF-I a causa della patologica ipersecrezione di GH da parte del tumore ipofisario, aggravata dalla concomitante iperinsulinemia.

\section{Come agiscono gli analoghi della somatostatina}

Gli analoghi della somatostatina (SA) si legano ai recettori della somatostatina i cui sottotipi 2 (sst2) e 5 (sst5) sono i mediatori principali delle azioni dei LA-SMSA oggi disponibili. In considerazione dell'espressione dei sst2 e sst5 nelle cellule somatrotrope, l'ipersecrezione patologica di GH può essere inibita dai SA con conseguente riduzione della produzione epatica di IGF-I. Quando tale riduzione è sufficiente a normalizzare i livelli di IGF-I, il trattamento è considerato adeguato [7]. Peraltro, i LA-SMSA si legano ai sottotipi sst2 e sst5 anche nelle isole pancreatiche, provocando una riduzione della secrezione di glucagone e diinsulina e determinando, occasionalmente, un peggioramento del controllo glicemico nei pazienti trattati. Inoltre, una soppressione della secrezione insulinica da parte dei LA-SMSA risulta anche selettivamente in una resistenza epatica al GH, il che riduce la produzione epatica di IGF-I [5]. Pertanto, la susseguente riduzione della IGF-I circolante non riflette, necessariamente, l'attività del GH nei tessuti periferici.

Un effetto soppressivo dei SMSA (GH-indipendente) sulle IGF-I circolanti è stato documentato in due studi nell'uomo [8]. Entrambi gli studi prevedevano la somministrazione di octreotide per 7 giorni durante trattamento con $\mathrm{GH}$ in pazienti con deficit di GH (GHD). È stata osservata una significativa riduzione del 16-18\% delle IGF-I circolanti con riduzione concomitante dell'insulinemia associata ad elevati livelli di IGFBP-1 [8].

È quindi plausibile, nel contesto della malattia acromegalica, che la normalizzazione dei livelli circolanti di IGFI durante trattamento con LA-SMSA non implichi, necessariamente, il controllo della malattia nei tessuti periferici,

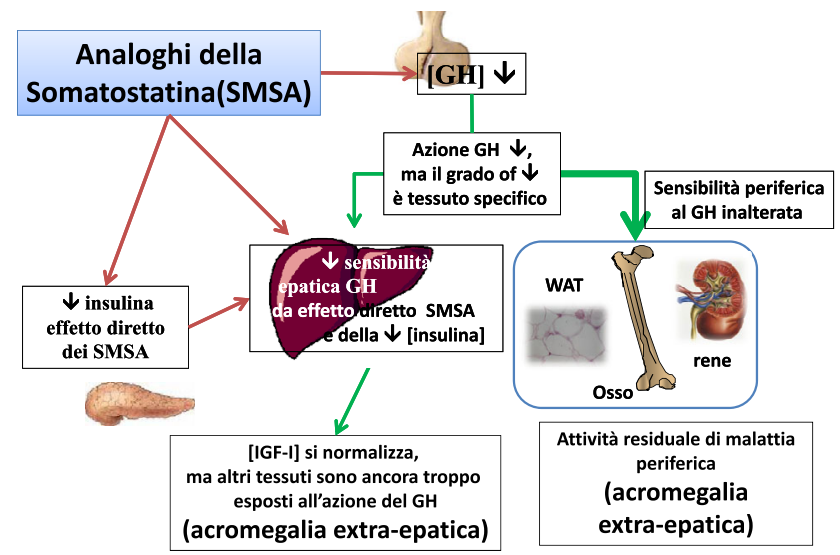

(a)

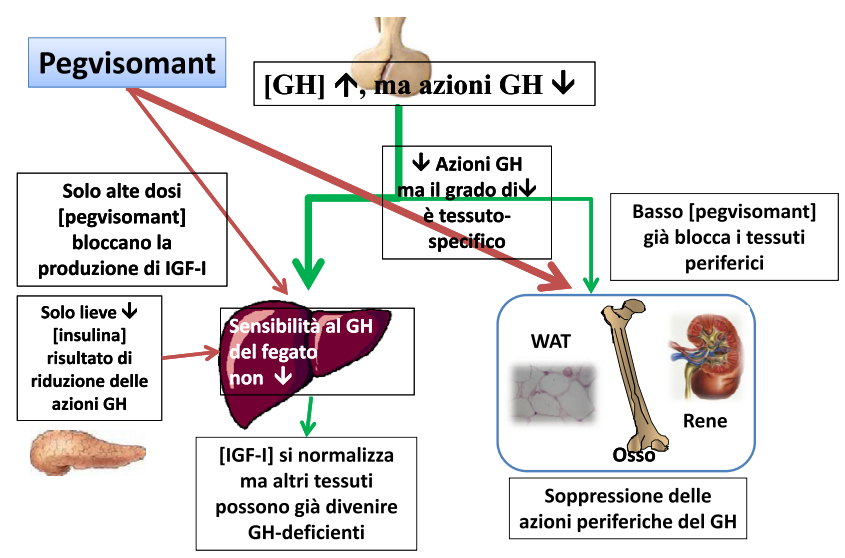

(b)

Fig. 1 a Effetti degli analoghi della somatostatina (SMSA) in acromegalici sensibili a tali composti. Le frecce rosse indicano effetti inibitori, le verdi indicano effetti stimolatori, mentre lo spessore delle frecce indica il livello di inibizione. WAT, tessuto adiposo bianco (modificata da [9]); b Effetti del pegvisomant negli acromegalici. Le frecce rosse indicano effetti inibitori, lo spessore delle frecce indica il livello di inibizione. WAT, tessuto adiposo bianco (modificata da [9])

configurando cioè una condizione per la quale abbiamo proposto la denominazione di acromegalia extra-epatica [9], i cui meccanismi sono schematicamente rappresentati nella Fig. 1a.

In assenza di appropriati parametri bioumorali non è agevole dimostrare se l'acromegalia extra-epatica sia un'entità clinico-nosografica piuttosto che un problema semantico, ma è degno di nota il rilievo di una scarsa qualità di vita in pazienti trattati con LA-SMSA, nonostante la normalizzazione dei livelli circolanti di IGF-I [10].

Rubeck et al. hanno valutato, in acromegalici trattati soltanto chirurgicamente o con LA-SMSA, differenti biomarcatori e lo stato di salute globale [11]. Le IGF-I totali e biologicamente attive erano simili; nel gruppo trattato con LASMSA i livelli di GH in risposta al carico di glucosio risultavano più elevati rispetto ai pazienti sottoposti a chirurgia mentre lo stato di salute specifico della malattia risultava migliore nei pazienti operati [11]. Nonostante i livelli di IGF-I 
fossero simili e normalizzati in ambo i gruppi, il trattamento con LA-SMSA si associava a minore soppressione del GH e meno evidente riduzione dei sintomi, rispetto alla chirurgia, supportando il concetto di acromegalia extra-epatica.

\section{Come agiscono gli antagonisti del recettore del GH}

Come sopra accennato, l'antagonista del recettore del GH, PEGV, blocca in modo competitivo i recettori del GH in tutti i tessuti. Più alta è la quantità di GH disponibile, maggiore sarà la quantità di PEGV necessaria a bloccare efficacemente l'azione del GH [1]. Il PEGV, comunque, non è egualmente efficace nel bloccare l'azione del GH in ogni tessuto. Il tessuto adiposo, il rene e il muscolo scheletrico richiedono meno PEGV rispetto al fegato che, a sua volta, necessita di maggiori quantità di PEGV per ridurre la produzione di IGF-I. A ulteriore conferma di questo è stato di recente riportato che la somministrazione di PEGV a breve termine in persone sane può sopprimere la lipolisi senza influenzare $\mathrm{i}$ livelli circolanti o locali di IGF-I. È quindi possibile che il trattamento con PEGV nell' acromegalia sia condizionato da differenze tessuto-specifiche in maniera dose-dipendente. In particolare, è possibile che la soppressione periferica dell'attività del GH si ottenga prima della normalizzazione della produzione epatica di IGF-I. Tale condizione, che si verifica durante la terapia con PEGV, potrebbe essere definita acromegalia epatica (Fig. 1b) che rappresenterebbe in sostanza il reciproco della condizione di acromegalia extra-epatica, che si realizza durante la terapia con LA-SMSA [9], sebbene attualmente non esista chiara evidenza nell'uomo che possa indicare con certezza che l'acromegalia epatica insorga durante monoterapia con PEGV.

\section{Lezioni dal diabete di tipo 1 e di tipo 2}

Gli effetti del ripristino dei livelli portali di insulina sulle IGF-I circolanti sono stati studiati nel diabete di tipo 1 . Studi sperimentali hanno dimostrato che una riduzione dei livelli di GH si osserva solo in seguito alla somministrazione portale di insulina, in grado di normalizzare i livelli di IGF-I, sebbene l'evidenza clinica dimostri che pazienti diabetici in trattamento convenzionale con insulina presentino generalmente bassi livelli di IGF-I e aumentati livelli di GH. Wurzburger et al. hanno studiato, nel diabete tipo 1 , i livelli di IGF-I GH-stimolati. I pazienti sono stati divisi in due gruppi, uno C-peptide negativo senza residua attività $\beta$-cellulare, il secondo C-peptide positivo con attività $\beta$-cellulare conservata [12]. L'aumento dei livelli circolanti di IGF-I veniva osservato soltanto nei pazienti con attività $\beta$-cellulare residua [12]. Esistono diverse analogie fra i pazienti con diabete di tipo 1 e gli acromegalici trattati con LA-SMSA: entrambi esibiscono elevata attività sistemica del GH con relativa resistenza epatica dovuta alla riduzione dell'insulina portale, sebbene i diabetici di tipo 1 presentino bassi livelli di IGF-I mentre gli acromegalici trattati con LA-SMSA posseggono livelli di IGF-I normali o elevati.

Ci sono anche analogie fra il diabete di tipo 2 e l'acromegalia trattata con PEGV: in entrambe le condizioni si osserva, infatti, una bassa attività sistemica del GH in presenza di sensibilità epatica relativamente aumentata per effetto di livelli portali di insulina normali o elevati.

\section{Terapia combinata con analoghi della somatostatina e Pegvisomant}

Numerosi articoli hanno presentato dati sulla terapia combinata con LA-SMSA e PEGV, perlopiù focalizzati su pazienti non controllati dalla mono-terapia con LA-SMSA [13]. È tuttavia possibile che il trattamento in combinazione possa esercitare effetti benefici indipendentemente dal controllo delle IGF-I, come chiaramente dimostrato da Neggers et al. [14]. Questo studio aveva infatti ipotizzato che la somministrazione settimanale di $40 \mathrm{mg}$ di PEGV potesse migliorare la qualità della vita $(\mathrm{QoL}) \mathrm{e}$ vari parametri metabolici in pazienti acromegalici con normali concentrazioni di IGF-I (aggiustate per età) durante terapia con LA-SMSA. Nello studio, 20 acromegalici sono stati trattati con PEGV o placebo per due periodi consecutivi di 16 settimane separate da 4 settimane di wash out. L'efficacia è stata determinata in base a specifiche modificazioni della QoL alla fine di ogni periodo di trattamento rispetto alle condizioni basali. La QoL è stata valutata in base al questionario Acromegaly Quality of Life Questionnaire (AcroQoL) e al Patient-assessed Acromegaly Symptom Questionnaire (PASQ). È stato interessante rilevare che AcroQoL è migliorata dopo l'aggiunta di PEGV, che ha parimenti migliorato significativamente il PASQ (in particolare i singoli quesiti riguardanti sudorazione, ingrandimento dei tessuti molli e complessivo stato di benessere). $\mathrm{Al}$ contrario, non si è osservata alcuna modificazione significativa dei livelli di IGF-I durante la somministrazione di PEGV. Considerando che i valori normali corretti per l'età hanno range relativamente ampi, è possibile che alcuni pazienti abbiano valori di IGF-I compresi nel range normale per la popolazione generale ma troppo alti per il singolo individuo. Il PEGV potrebbe pertanto avere indotto un breve e transitorio declino di IGF-I non documentabile al momento del prelievo di sangue 7 giorni dopo l'iniezione del peptide, ma in grado di indurre i propri effetti clinici, come evidenziato dai questionari.

Dunque, basse dosi di PEGV sono in grado di migliorare i segni e i sintomi extra-epatici di acromegalia senza influenzare la produzione epatica di IGF-I, in accordo con l'ipotesi di acromegalia extra-epatica. È degno di nota che il massimo miglioramento della QoL è stato osservato in pazienti 
che rispondevano al PEGV anche in termini di riduzione della ritenzione di liquidi [14]. Resta peraltro da chiarire se gli stessi effetti favorevoli possano essere ottenuti aumentando la dose di LA-SMSA.

\section{Considerazioni finali e indirizzi futuri}

Gli analoghi della somatostatina sono ormai riconosciuti come farmaci sicuri ed efficaci nel trattamento dell'acromegalia, sebbene un adeguato controllo della malattia non sia sempre raggiunto. Con la recente introduzione in terapia del PEGV è oggi possibile ottenere il controllo biochimico della malattia nella maggior parte dei pazienti.

È quindi giunto il momento per un'analisi critica dell'appropriato metodo di valutazione dei risultati della terapia con queste due diverse modalità. È ipotizzabile che la misura dell'IGF-I circolante non sia, necessariamente, il marcatore biologico più affidabile dell' attività di malattia. Gli analoghi della somatostatina hanno infatti tre effetti tessuto-specifici. Il primo è la riduzione della secrezione di GH da parte del tumore ipofisario, il secondo la riduzione della secrezione pancreatica di insulina e il terzo la riduzione della produzione epatica di IGF-I, che può condurre alla normalizzazione dei suoi livelli circolanti, in contrasto con l'insufficiente controllo dell'attività della malattia nei tessuti periferici.

La combinazione di tali effetti può condurre a una normalizzazione dei livelli circolanti di IGF-I in presenza di una residua attività periferica di malattia, cioè l'acromegalia extra-epatica. Se questa condizione abbia un significato clinico e se possa essere controllata dal semplice aumento della dose di LA-SMSA merita di essere oggetto di un trial clinico controllato che paragoni gli effetti dei LA-SMSA in pazienti randomizzati per la dose di LA-SMSA e per i livelli di IGF-I o GH.

L'impiego del PEGV può, peraltro, diventare problematico in quanto associato a ulteriore incremento dei livelli di $\mathrm{GH}$ e, inoltre, alcune evidenze suggeriscono che il PEGV possa indurre un blocco significativo dell'azione periferica del GH prima di bloccarne l'attività epatica. In tale contesto è anche degno di attenzione il fatto che le dosi di PEGV necessarie per il controllo della malattia sono soggette ad ampie variazioni fra paziente e paziente. Saranno pertanto necessari, in aggiunta all'IGF-I, nuovi biomarcatori in grado di valutare le risposte individuali al trattamento e sarà importante stabilire l'utilità del dosaggio del PEGV circolante.

Il fatto che LA-SMSA e PEGV esercitino effetti soppressivi complementari sull'asse GH-IGF-I rende la terapia combinata un'opzione interessante. Vi è infatti evidenza che la terapia combinata sia superiore alla mono-terapia con LA-SMSA in termini di controllo dell'omeostasi glicidica [13] e della QoL specifica per la malattia acromegali-

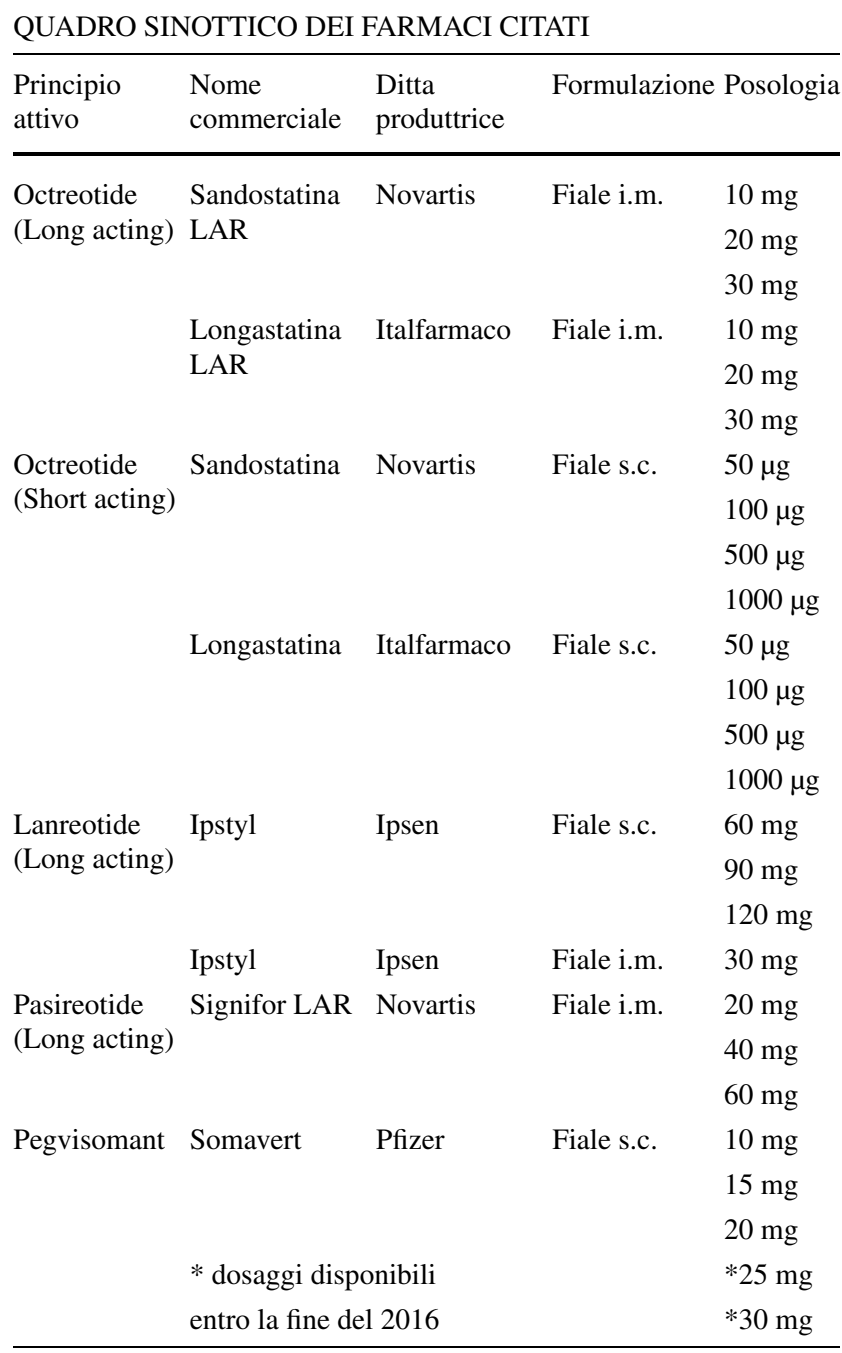

Per l'elenco completo dei farmaci citati e le relative schede tecniche si rimanda al prontuario dei farmaci

ca [14]. Quest'ultima osservazione indica, pertanto, l'utilità della valutazione routinaria della $\mathrm{QoL}$ in corso di terapia.

In considerazione di quanto esposto in questa rassegna, appare evidente come sia cruciale l'individuazione di nuovi biomarcatori (genomici, metabolomici, proteomici o altro) in grado di integrare, idealmente, l'attività epatica e periferica della malattia acromegalica.

Conflitto di interesse L'autore Aart J. van der Lelij dichiara assenza di conflitti di interesse.

Consenso informato Lo studio presentato in questo articolo non ha richiesto sperimentazione umana.

Studi su animali L'autore non ha eseguito studi su animali per la redazione di questo articolo.

Open Access This article is distributed under the terms of the Creative Commons Attribution 4.0 International License (http://creative 
commons.org/licenses/by/4.0/), which permits unrestricted use, distribution, and reproduction in any medium, provided you give appropriate credit to the original author(s) and the source, provide a link to the Creative Commons license, and indicate if changes were made.

\section{Bibliografia}

1. van der Lely AJ, Hutson RK, Trainer PJ et al (2001) Long-term treatment of acromegaly with pegvisomant, a growth hormone receptor antagonist. Lancet 358:1754-1759

2. List EO, Palmer AJ, Berryman DE et al (2009) Growth hormone improves body composition, fasting blood glucose, glucose tolerance and liver triacylglycerol in a mouse model of diet-induced obesity and type 2 diabetes. Diabetologia 52:1647-1655

3. Yang CW, Striker LJ, Pesce C et al (1993) Glomerulosclerosis and body growth are mediated by different portions of bovine growth hormone. Studies in transgenic mice. LabInvest 68:62-70

4. Moller N, Vendelbo MH, Kampmann U et al (2009) Growth hormone and protein metabolism. Clin Nutr 28:597-603

5. Leung KC, Doyle N, Ballesteros M et al (2000) Insulin regulation of human hepatic growth hormone receptors: divergent effects on biosynthesis and surface translocation. J Clin Endocrinol Metab $85: 4712-4720$

6. Jorgensen JO, Krag M, Jessen N et al (2004) Growth hormone and glucose homeostasis. Horm Res 62(Suppl 3):51-55

7. Giustina A, Bonadonna S, Bugari G et al (2009) High-dose intramuscular octreotide in patients with acromegaly inadequa- tely controlled on conventional somatostatin analogue therapy: a randomised controlled trial. Eur J Endocrinol 161:331-338

8. Pokrajac A, Frystyk J, Flyvbjerg A, Trainer PJ (2009) Pituitaryindependent effect of octreotide on IGF1 generation. Eur J Endocrinol 160:543-548

9. Neggers SJ, Kopchick JJ, Jorgensen JO, van der Lely AJ (2011) Hypothesis: extra-hepatic acromegaly: a new paradigm? Eur J Endocrinol 164:11-16

10. Bonapart IE, van Domburg R, Ten Have SM et al (2005) The "bioassay" quality of life might be a better marker of disease activity in acromegalic patients than serum total IGF-I concentrations. Eur J Endocrinol 152:217-224

11. Rubeck KZ, Madsen M, Andreasen CM et al (2010) Conventional and novel biomarkers of treatment outcome in patients with acromegaly: discordant results after somatostatin analog treatment compared with surgery. Eur J Endocrinol 163:717-726

12. Wurzburger MI, Prelevic GM, Sonksen PH et al (1995) Effect of recombinant human growth hormone treatment on insulin-like growth factor (IGF-I) levels in insulin-dependent diabetic patients. Acta Diabetol 32:131-134

13. Jorgensen JO, Feldt-Rasmussen U, Frystyk J et al (2005) Cotreatment of acromegaly with a somatostatin analog and a growth hormone receptor antagonist. J Clin Endocrinol Metab 90:5627-5631

14. Neggers SJ, van Aken MO, de Herder WW et al (2008) Quality of life in acromegalic patients during long-term somatostatin analog treatment with and without pegvisomant. J Clin Endocrinol Metab 93:3853-3859 